\title{
FORMACIÓN Y ORIENTACIÓN PARA EL EMPRENDIMIENTO. LO QUE DICEN LA BIBLIOMETRÍA Y LOS EMPRENDEDORES NOVELES
}

\section{TRAINING AND GUIDANCE FOR ENTREPRENEURSHIP. WHAT BIBLIOMETRICS AND NOVICE ENTREPRENEURS SAY}

\author{
Davinia Palomares-Montero ${ }^{1}$ \\ María José Chisvert-Tarazona \\ Universidad de Valencia, España \\ Magdalena Suárez-Ortega \\ Universidad de Sevilla, España
}

\section{RESUMEN}

El actual contexto socioeconómico obliga a realizar una revisión profunda del sentido del trabajo en la población. En momentos de escasez de oportunidades laborales y de redefinición del sentido y el significado del trabajo muchas personas consideran la opción del autoempleo como alternativa al desempleo y la precariedad laboral. Sin embargo, informes oficiales muestran altas tasas de abandono de los proyectos empresariales en sus primeros años. Con la intención de conocer las razones de esta situación, se centra la atención en analizar el papel que juega la orientación profesional en las fases iniciales del emprendimiento para favorecer una reflexión profunda sobre los motivos y las motivaciones que llevan a emprender. Para ello, mediante el análisis bibliométrico se analiza el tratamiento que la comunidad científica ha realizado de la

\footnotetext{
${ }^{1}$ Correspondencia: Davinia Palomares-Montero: Avenida Blasco Ibáñez, 30. 46010, Valencia (España). Correo-e: davinia.palomares@uv.es, web: https://www.uv.es/didorg
} 
orientación profesional como impulsora del emprendimiento. El método Delphi completa la investigación, que permite una aproximación a la esfera profesional, a través de las opiniones de emprendedores noveles. La triangulación de estas dos metodologías permite generar un espacio de análisis que permite redefinir el modo de actuación de la orientación profesional frente al emprendimiento. Los resultados ponen de relieve la necesidad de avanzar en el diseño de propuestas orientadoras y formativas que motiven al emprendimiento desde una visión integral.

Palabras clave: Emprendimiento, orientación profesional, proyecto profesional, bibliometría, método Delphi.

\section{ABSTRACT}

The current socioeconomic definition requires a deep revision of the meaning of work in the population. When job opportunities are limited and the meaning of work is redefining itself, many people consider the option of self-employment as an alternative to unemployment and job insecurity. However, official reports show high rates of abandonment of business projects in their early years. To understand the reasons for this high level of business failure, we focus our attention on analysing the role that professional guidance plays in the initial phases of entrepreneurship to encourage a deep reflection on the reasons and motivations that lead to undertake a business project. We apply bibliometric analysis to analyse the scientific community's treatment of professional orientation as a promoter of entrepreneurship. We further complete our investigation with the Delphi method, which allows us to approach the real sphere: the opinion of novice entrepreneurs. Using these two methodologies allows generating a space of analysis that permits to redefine the way of acting of the career guidance in front of the entrepreneurship. The results highlight the need to advance the design of guiding and training proposals that motivate entrepreneurship from a holistic point of view.

Keywords: Entrepreneurship, career guidance, professional project, bibliometrics, Delphi method

\section{Introducción}

El siglo XXI enfrenta a su ciudadanía, quizás más que en otras épocas, a la revisión permanente de sus proyectos profesionales y de vida (Chisvert-Tarazona, 2014). Las sociedades occidentales están marcadas en la actualidad por la desaparición de certezas, por el relativismo, también por una cierta desesperanza. El desmantelamiento progresivo del Estado de Bienestar que sufre Europa, justificado en el discurso institucional por la fragilidad de la economía en un mundo globalizado, suprime certezas que habían permitido la consolidación de derechos fundamentales como la educación, la vivienda o la salud. Sociedades instaladas en la incertidumbre, en la modernidad líquida (Bauman, 1999; Morin, Ciurana y Mota, 2002; Beck, 2006), en las que sus poblaciones han perdido la confianza en las instituciones. Esta situación, agravada con la crisis económica iniciada a finales de la primera década del siglo XXI, y que continúa haciendo mella una década después, es especialmente difícil en países del sur de Europa donde se evidencian tasas de desempleo persistentemente altas, de mayor duración y que afectan especialmente a la población más joven (European Commission, 2014). 
Este contexto explica la revisión del sentido y significado del trabajo entre la población, cuya realidad sociolaboral se caracteriza por trayectorias profesionales, en muchos casos, truncadas. Las instituciones gubernamentales, nacionales e internacionales, valoran el autoempleo como una fórmula apropiada de superación de la precariedad laboral y el desempleo (Perren y Jennings, 2005; Pinto, 2005; Cumming, Sapienza, Siegel y Wright, 2009). Sin embargo, esta circunstancia ha despertado cierta desconfianza hacia un discurso institucional que traslada la responsabilidad sobre quienes en última instancia deciden emprender, obviando responsabilidades gubernamentales o empresariales. Se trata de una mirada mercantilizada e individualizada del emprendimiento entendido como descubrimiento, evaluación y explotación de una oportunidad de negocio dirigida a ofrecer a terceros un producto o servicio que conlleva considerar a la persona emprendedora como aquella capaz de valorar el riesgo y tomar conciencia de las oportunidades y necesidades de innovación (Shane y Venkataraman, 2000).

A pesar de esta desconfianza, las condiciones del contexto han convertido el autoempleo en una opción más habitual, aunque no siempre es producto de la identificación de una solución a un problema o necesidad, propio de un emprendimiento por oportunidad. En muchos casos se opta por el autoempleo como alternativa ocupacional ante la falta de opciones de contratación satisfactorias; se trata de un emprendimiento por necesidad. El Informe GEM muestra la evolución de este tipo de emprendimiento cuando, para el caso de España, sitúa en 2008 un 14.8\% de emprendimiento por necesidad, mientras que en el año 2016 éste asciende hasta el 26.0\% (Global Enterpreneurships Monitor, 2017).

En cualquier caso e independientemente del motivo por el que se decide emprender, la intención emprendedora no ha sido una cuestión muy extendida ni priorizada en el contexto español. El Informe GEM (Global Enterpreneurships Monitor, 2017) advierte que durante 2016 España se posicionó por debajo de la media de otras economías basadas en la innovación en cuanto al porcentaje de emprendimiento potencial (6.1\% frente al $18.5 \%$ de media), el nivel de actividad emprendedora en fase inicial $(5.2 \%$ frente al $9.1 \%$ de media) y el porcentaje de empresariado consolidado (6.2\% frente al $6.7 \%$ de media). En este contexto, se considera la necesidad de entender la orientación profesional como estrategia y servicio de apoyo y estímulo para el desarrollo de la carrera emprendedora, debiéndose potenciar un sistema integral que ponga en relación el conjunto de experiencias, background y trayectoria personal, con el diseño y la gestión de un proyecto profesional emprendedor. Esta fue una carencia detectada al inicio del proyecto en el que se enmarca este trabajo, analizada en conexión con el estado sociolaboral y el sistema de formación y orientación profesional en su momento, y de lo que había escasa literatura científica al respecto en nuestro campo y en otros afines. Desde estos presupuestos que motivaron el estudio, en este trabajo se reivindica el papel crucial que debe ejercer la orientación profesional en el diseño y la gestión de proyectos profesionales emprendedores, específicamente en contextos sociolaborales y en relación con el trabajo autónomo y la generación de tejido productivo, capital humano e innovación social y empresarial, reivindicación clave en el Proyecto I+D en el que se enmarca este estudio (Financiado por el Ministerio de Economía y Competitividad), línea en la que venimos trabajando desde final de 2012.

Concretamente, el objetivo de este trabajo es conocer, a través de un análisis bibliométrico, el estado del arte respecto de las investigaciones científicas o experiencias formativas desarrolladas sobre orientación profesional para el emprendimiento en España, así como poner en relación los resultados obtenidos con el conocimiento aportado por emprendedores noveles sobre sus requerimientos formativos. Se trata de generar un espacio de estudio que permita la revisión de las pautas de actuación de la orientación profesional frente al emprendimiento. Desde los servicios de orientación y asesoramiento se podría trabajar en un enfoque y metodología integradora de la carrera, apoyando la construcción de proyectos profesionales y de vida coherentes y funcionales que posibiliten la articulación de las necesidades inmediatas con las metas de autorrealización (Patton y McMahon, 2006; Sánchez-García y Suárez-Ortega, 2017). 


\section{El emprendimiento y la orientación profesional, una extraña pareja}

Según la OCDE (2001), el emprendimiento es un fenómeno asociado a la actividad humana en su búsqueda de generación de valor, de creación o expansión económica, a través de la identificación y explotación de nuevos productos, procesos o mercados. Esta definición, entre las más ampliamente aceptadas, parte de las teorías subjetivistas, obedece a las reglas de la ciencia económica y a una visión racional y utilitaria de los proyectos empresariales. Sin embargo, otras perspectivas teóricas aportan nuevos elementos a la comprensión de la iniciativa emprendedora y se extienden a campos menos orientados a los factores económicos (Pfeilstetter, 2011; Palomares-Montero y Chisvert-Tarazona, 2015). En esta línea, la Organización Internacional del Trabajo define el emprendimiento como la capacidad de reconocer una oportunidad para crear valor y actuar para aprovecharla, más allá de si se trata de la formación de una nueva entidad empresarial (Schoof, 2006). La propuesta de Manso y Thoilliez (2014) define la iniciativa emprendedora como una competencia clave que introduce cambios prósperos en la evolución de cada uno, posibilita la adaptación positiva y enriquecedora a los cambios y a las oportunidades que se presentan en el entorno y se responsabilizan de las acciones propias desarrollando y evaluando estrategias para alcanzar el éxito. Las autoras de este artículo comparten esta mirada más abierta del emprendimiento, siendo conscientes de que representa una transición dirigida a la creación de valor, que requerirá de una toma de decisiones pausada, de un trabajo de conocimiento del entorno, pero también de autoconocimiento, dirigido a evaluar la idoneidad de la acción emprendedora. Autores como Manso y Thoilliez (2014) o el propio Parlamento Europeo (2006) proponen que la competencia de emprendimiento debe considerar varias dimensiones: la cognitiva, la instrumental y la actitudinal. La cognitiva, que permita identificar las oportunidades disponibles para la mejora personal, las posibilidades de autoempleo, comprender el contexto económico-laboral o promover la superación de oportunidades y retos. La instrumental, próxima al diseño y la gestión de proyectos, la planificación, organización, análisis, liderazgo, comunicación, gestión, ejecución, evaluación, o el trabajo individual y cooperativo en equipos. Y la actitudinal, relativa a la proactividad, la iniciativa, la independencia y el liderazgo, la innovación y la creatividad, la conciencia ética en el contexto institucional y la sostenibilidad e impacto ecológico.

La orientación profesional, considerada bisagra posibilitadora de transiciones, tiene la obligación de ofrecer respuestas integrales a las necesidades que acompañan al emprendimiento actual. Si se atiende a las dimensiones anteriores, cognitiva-instrumental-actitudinal, se evidencia la relevancia de la orientación profesional en el avance y consolidación de esta competencia y su importancia para aportar formación y asesoramiento en cada una de las dimensiones. De hecho, Suárez-Ortega, Sánchez-García y García-García (2016) evidencian, en un estudio realizado sobre buenas prácticas y necesidades de mejora de los servicios de orientación, que el $32.0 \%$ de las personas que acuden a estos servicios perciben necesidades de formación/orientación sobre autoempleo. Además, en un estudio posterior se ha puesto de manifiesto que en la gestión de la carrera emprendedora operan dos núcleos competenciales que deben actuar en equilibrio y coherencia: (1) una habilidad de carácter cognitivo-reflexivo sobre las propias metas y reflexiones; y (2) una habilidad de carácter más práctico y aplicado (Suárez-Ortega y Sánchez-Ortega, 2017). Este ejercicio de práctica reflexiva puede realizarse con el apoyo de profesionales de la orientación. De hecho, resultados en esta línea se aportan en el estudio de Soto-González, Suárez-Ortega y Ortega-Leal (en prensa).

En su historia reciente, los servicios de orientación profesional han tendido a aproximarse al emprendimiento sin generar espacios de reflexión sobre el proyecto profesional y de vida de la persona que decide emprender. En muchos casos el proceso orientador se ha reducido al análisis de viabilidad de la idea empresarial. Sin embargo, la orientación profesional debe realizarse desde un enfoque holístico e integral que amplía sus requerimientos. La intervención en este campo 
requiere de un relato relativo al proyecto de vida; en palabras de Savickas $(2005,2012)$, del diseño de vida. Se contempla la orientación profesional a partir de premisas sobre el yo o la identidad que se concentra en las posibilidades contextuales, generando progresos dinámicos, no lineales, a través de los cuales una persona, también una persona emprendedora, "construye una carrera subjetiva, una historia sobre su vida laboral" (M. Savickas, 2012, p. 90).

Paralelamente, las políticas europeas despliegan su influencia en los Estados miembros de forma cada vez más significativa, también en lo que respecta a la formación para el emprendimiento (Bulmer y Lequesne, 2013; Kumar y Parveen, 2013). En esta línea, la Estrategia de Lisboa lanzó acciones dirigidas a ampliar la cooperación de los Estados miembros en materia de enseñanza (Consejo Europeo, 2000), definiendo un marco europeo de referencia dirigido a concretar las competencias clave del aprendizaje permanente (Parlamento Europeo, 2006; Parlamento y Consejo Europeo, 2006) e incluyendo, entre otras, la competencia "Iniciativa y Espíritu Emprendedor". La introducción de su formación en el sistema educativo formal parte en España de una estrategia de planificación integrada, de un enfoque curricular transversal y obligatorio y de la disposición de directrices y materiales de orientación al profesorado (Manso y Thoilliez, 2015). Se trata sin duda de un avance cualitativo en el desarrollo de la competencia emprendedora entendida como iniciativa, capacidad de innovación, actitud positiva ante el cambio $y$, al mismo tiempo, responsabilidad de las acciones propias.

En este sentido, el nivel formativo también ha sido analizado en muchos estudios como condicionante para el emprendimiento aunque con resultados dispares. Mientras que Fairlie y Robb (2009) observan la existencia de una relación positiva entre el nivel educativo, el emprendimiento y los resultados económicos obtenidos, Grilo e Irigoyen (2006), que estudian el efecto que tiene el género y el nivel de estudios en el espíritu emprendedor latente y real, concluyen que ambos factores no inciden de forma significativa en el hecho de emprender. Dado que existen posicionamientos diversos respecto a la incidencia de los aspectos formativos en el proceso emprendedor, en el presente trabajo se quiere dar un paso más y, partiendo de una postura favorable respecto a la formación como factor relevante que promueve el emprendimiento, se presenta una revisión bibliométrica de la literatura que avanza sobre este aspecto y la opinión que emprendedores noveles manifiestan sobre la necesidad de contar con esta formación. Así, siguiendo a Sánchez-García y Suárez-Ortega (2017), se parte de la idea de que la formación para el emprendimiento no sólo requerirá del asesoramiento en términos económicos, sino que será necesario contar con un modelo holístico con enfoque por competencias (Patton y McMahon, 2006; Savickas, 2013) que integre acciones orientadoras individualizadas centradas en el desarrollo y gestión de la carrera emprendedora, desde el análisis pormenorizado de los perfiles competenciales. A continuación, se muestra cómo la literatura científica ha avanzado en este sentido y lo que emprendedores noveles opinan al respecto del objeto de estudio.

\section{Metodología}

El estudio que se presenta, por la complejidad del objetivo de investigación que persigue, emplea un análisis multimetodológico para tratar de responder de forma holística al tema de estudio. Por un lado, se aplica una revisión bibliográfica para identificar estudios relevantes, valorando su calidad, sobre la orientación profesional para el emprendimiento. Por otro lado, se aplica una metodología descriptiva pues no se pretende construir teoría generalizable a otros contextos sino describir un fenómeno particular como es la orientación para el emprendimiento. Ambas metodologías se instrumentalizan en dos fases. En la primera fase se desarrolla el análisis bibliométrico como técnica elegida para realizar la revisión bibliográfica. En la segunda fase se 
lleva a cabo el método Delphi como técnica para conocer de forma más cualitativa y descriptiva las necesidades formativas que faciliten la orientación para el emprendimiento.

\section{Análisis bibliométrico}

Como se ha comentado, una de las finalidades de la investigación es realizar un análisis bibliométrico que permita conocer todo aquello que se ha publicado sobre orientación y formación para el emprendimiento en España. La bibliometría se define por su rigurosidad ya que posee un objetivo de investigación concreto, una estrategia de búsqueda específica e incluye la localización sistemática de toda la información disponible sobre el tema estudiado (Giménez-Espert y PradoGascó, 2012; Dorta-González y Ramírez-Sánchez, 2014).

Para la realización del estudio se seleccionó la base de datos Science Citation Index Expanded de la Web of Science (WOS) de Thomson Reuters, ampliamente utilizada en estudios bibliométricos. Se analizaron los documentos publicados en las revistas incluidas en la Colección Principal del Web of Science sin establecer límite temporal pero sí un filtro para el tipo de documento seleccionando exclusivamente los trabajos científicos publicados como "articles" dado que el interés se centraba en analizar los trabajos de impacto publicados en revistas de reconocido prestigio. De los primeros resultados se decidió seleccionar aquellos pertenecientes a las categorías "Education Educational Research", "Education Scientific Disciplines" y "Management", próximas al área de estudio objeto de este trabajo.

En este proceso es extremadamente importante realizar una correcta elección de los términos de búsqueda, de los campos y de la ecuación de búsqueda dado que WOS "no utiliza vocabularios temáticos controlados del tipo de los tesauros" (E. Delgado López-Cózar y A. Fernández Cano, 2002, p. 611). En nuestro caso, tras consultar el thesauro "Educational Resources Information Center -ERIC", se optó por los vocablos "guidance" y "entrepreneurship", siendo la ecuación de búsqueda empleada la siguiente: guidance AND entrepreneur*. El operador de truncamiento de sufijo $\left({ }^{*}\right)$ permite recuperar las diferentes terminaciones del vocablo elegido. El campo elegido para la búsqueda fue el "Tema" que acoge al título, el resumen y las palabras clave, de hecho es el campo predeterminado que propone WOS. Los datos bibliométricos se descargaron en Histcite la primera semana de Enero de 2018.

\section{Metodología interpretativa: Método Delphi}

En esta sección, se presenta la metodología que dará respuesta al segundo de los propósitos del trabajo: poner en relación los resultados obtenidos en la revisión bibliométrica con el conocimiento aportado por emprendedores noveles sobre sus requerimientos formativos. Para tal fin, se diseñó un instrumento que permitiera la aplicación del método Delphi. Esta técnica permite dar visibilidad a los resultados obtenidos y confirmar el grado de consenso sobre los mismos. El método Delphi permite establecer conclusiones de la investigación respecto a las necesidades de formación partiendo de la creación de una visión colectiva de personas expertas a partir tanto del consenso como del disenso (Landeta, 2002).

Concretamente se han aplicado dos rondas. En la primera, llevada a cabo en el mes de marzo de 2016, se mostraban varios bloques de preguntas abiertas que respondían a tres aspectos de formación/orientación para la puesta en marcha de un proyecto emprendedor: competencias, conocimientos y recursos de apoyo. En la segunda ronda, desarrollada en mayo de 2016, a partir del proceso de iteración anónima que evita que unos participantes puedan influir en las respuestas 
de los otros y que permite la reafirmación o rectificación de las opiniones de cada persona (Cabero e Infante, 2014), se obtuvo el posicionamiento final de los participantes con respecto a la utilidad de los aspectos mostrados en primera ronda. El número de personas participantes en primera ronda fue de 19 , habiendo una mortandad del $31.5 \%$ en segunda ronda y por tanto, participando 13 personas; número suficiente si se atiende a recomendaciones de Landeta (2002).

Las personas participantes son emprendedoras en transición y se caracterizan por ser: (1) emprendedoras potenciales, personas en edad de producir que han expresado su intención de emprender en los próximos tres años; (2) emprendedoras nacientes, personas con iniciativas que se hallan en la fase inicial en el desarrollo de su proyecto (máximo tres meses); o (3) emprendedoras nuevas, personas con iniciativas en fase de consolidación (entre 3 y 42 meses).

\section{Resultados}

\section{Orientación y emprendimiento: análisis de la productividad, pautas de colaboración y citaciones recibidas}

Para analizar los indicadores bibliométricos y las redes de citaciones respecto al campo objeto de estudio ha sido necesario utilizar dos softwares: Histcite (versión 10.12.6) y Bibexcel (versión 2011-02-03). Realizando dos fases en el análisis, en un primer momento se obtuvieron los indicadores bibliométricos básicos y a continuación el análisis de coocurrencia para establecer las relaciones entre los autores y las citaciones entre publicaciones científicas. Como indicadores básicos se muestran la evolución de la producción científica y de las citas recibidas en la WOS, así como las editoriales donde se publica sobre "guidance" y "entrepreneurship". Para el cálculo de las redes de colaboración se ha utilizado el programa Pajek (versión 1.28). El número de coocurrencias entre autores y entre citas no ha sido muy elevado lo que permite realizar una representación gráfica asequible y comprensible. Adicionalmente, se ha generado un mapa de densidad de citaciones con VOSviewer (versión 1.3.2).

El primer resultado obtenido en la búsqueda atendiendo a los vocablos "guidance" y "entrepreneurship" en la Colección Principal del Web of Science fue de 331 trabajos científicos. Tras aplicar el filtro por tipo de documento "article" el output se redujo a 162 trabajos, para finalmente analizar un total de 64 documentos una vez refinado por las categorías "Education Educational Research", "Education Scientific Disciplines" y "Management". Este dato permite manifestar que se trata de un tópico que ha sido escasamente estudiado desde el ámbito científico.

La evolución de la producción científica ha experimentado un incremento notable en el periodo que transcurre entre los años 2012 y 2017, que por otro lado es un periodo que coincide con el inicio y desarrollo del proyecto I+D en el que se enmarca este estudio, en los que se ha pasado de 19 publicaciones hasta el año 2011, a las 45 publicadas en esta franja temporal. Se podrían identificar tres tendencias de crecimiento en el periodo temporal resultante de la búsqueda: una primera fase, en la década de los noventa donde la producción sobre orientación para el emprendimiento es casi anecdótica; un segundo momento que experimenta un leve crecimiento, la primera década del siglo XXI, donde comienzan a aparecer nuevos trabajos al respecto; y un tercer periodo en el que cobra protagonismo la temática, la segunda década del siglo XXI, donde se publica el $78 \%$ de los trabajos recopilados en la búsqueda (véase Tabla 1). Esta tendencia no es replicable para el caso de las citas recibidas en WOS lo cual nos hace pensar que el número de artículos no guarda relación con el impacto de los mismos ya que, por ejemplo, el artículo 
publicado en 1993 ha obtenido un total de 410 citas en WOS, mientras que los cuatro artículos publicados en 2008 suman un total de 77 (incluso considerando la relación entre el número de citas y el año de publicación ya que cada año incluye las citas recibidas por todas las publicaciones de los años precedentes).

TABLA 1. Evolución de las publicaciones y las citas recibidas en WOS

\begin{tabular}{ccc}
\hline Año & No & Citas WOS \\
\hline 1982 & 1 & 13 \\
1993 & 1 & 410 \\
1997 & 1 & 1 \\
1998 & 1 & 82 \\
2001 & 2 & 368 \\
2004 & 1 & 2 \\
2005 & 1 & 33 \\
2007 & 1 & 163 \\
2008 & 4 & 77 \\
2009 & 1 & 1 \\
2010 & 2 & 98 \\
2011 & 3 & 41 \\
2012 & 5 & 107 \\
2013 & 4 & 63 \\
2014 & 4 & 19 \\
2015 & 13 & 36 \\
2016 & 10 & 20 \\
2017 & 9 & 1 \\
\hline Total & 64 & $\mathbf{1 5 3 5}$ \\
\hline & Fuente: Elaboración propia.
\end{tabular}

En lo referente a la revista donde se publican los trabajos se observa una gran dispersión. Un reducido número de revistas publican dos o más trabajos sobre orientación al emprendimiento, el resto, que concentra más del $50 \%$ de la producción, publica exclusivamente un artículo científico. Entre las primeras destacan principalmente revistas del área del "management", siendo solo dos las pertenecientes al área de "education" (véase Tabla 2), dato que asciende a seis si se observa el total de las 64 revistas identificadas. Destaca el hecho de que ninguna de ellas es española y los trabajos, a excepción de dos escritos en español y portugués respectivamente, están escritos en inglés.

TABLA 2. Revistas de publicación (al menos dos trabajos publicados)

\begin{tabular}{lcc}
\hline Revista & № & $\%$ \\
\hline Strategic Entrepreneurship Journal & 5 & 7.8 \\
Academy of Management Executive & 3 & 4.7 \\
Journal of Small Business Management & 3 & 4.7 \\
Small Business Economics & 3 & 4.7 \\
California Management Review & 2 & 3.1 \\
\hline
\end{tabular}




\begin{tabular}{lcc}
\hline Revista & № & $\%$ \\
\hline Education and Training & 2 & 3.1 \\
Eurasia Journal of Mathematics Science and Technology education & 2 & 3.1 \\
IEEE Transactions on Engineering Management & 2 & 3.1 \\
International Journal of Entrepreneurial Behavior and Research & 2 & 3.1 \\
International Small Business Journal & 2 & 3.1 \\
Management Decision & 2 & 3.1 \\
Management Research Review & 2 & 3.1 \\
Otras Revistas & 34 & 53.1 \\
\hline Total & 64 & 100 \\
\hline
\end{tabular}

Los datos han sido, en todos los casos, revisados para evitar duplicidades y el nombre de los autores y las autoras estandarizados para eliminar posibles errores de escritura en apellido y/o iniciales. En el caso de autores con nombre homogéneo o similar se ha utilizado el campo de "dirección del autor", teniendo que realizar alguna búsqueda puntual en Google ya que la base de datos WOS no ofrece esta información para todos los registros. De la búsqueda realizada se han identificado a un total de 175 autores, de los cuales solo dos (Fredrickson JW y Hambrick DC) han publicado más de un trabajo sobre orientación y emprendimiento. En la Figura 1 se puede observar el mapa de co-autorías que parece mostrar una amplia colaboración. De hecho, solo el $18 \%$ de los trabajos son firmados por un único autor, mientras que el $53 \%$ son firmados entre dos y tres autores y el $29 \%$ restante por cuatro o más autores.

FIGURA 1. Mapa de co-autorías

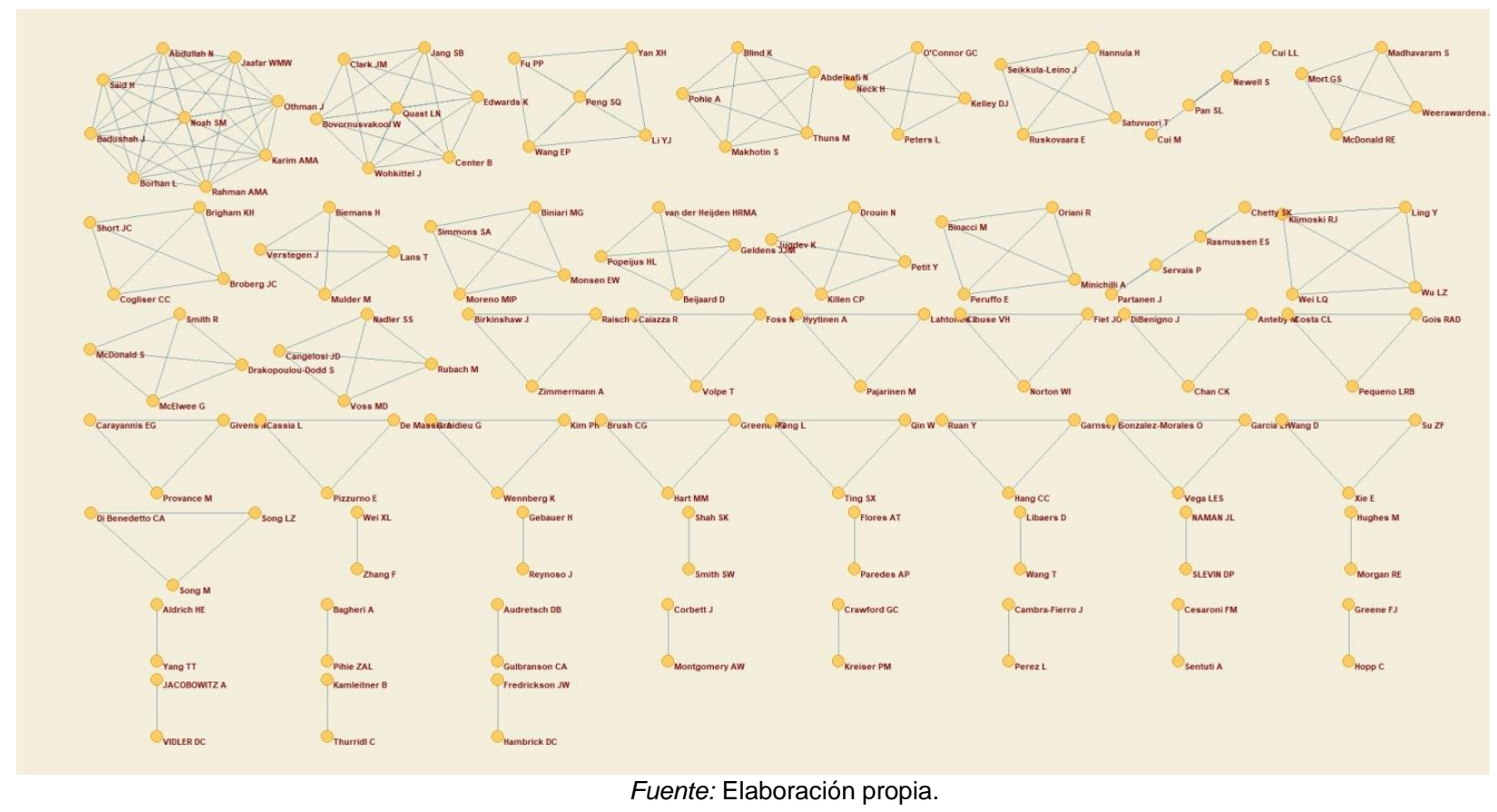


En la Figura 2 se puede observar la red de citaciones entre los artículos analizados. Conviene resaltar que las relaciones de las citas entre los trabajos analizados son bastante inconsistentes. De hecho, la red ha quedado compuesta por 30 autores. Se han encontrado tres núcleos grandes de citación (Figura 3). El primer grupo formado por autores que investigan aspectos relacionados con innovación, oportunidades y retos para emprender (Eisenhardt, Ndofor, Helfat), un segundo grupo central de citaciones de autores que citan trabajos relacionados con gestión de proyectos emprendedores (Sarasvathy, Cohen, Zahra) y finalmente un tercero que está orientado a actividades de emprendimiento relativos al medio ambiente (Baker).

FIGURA 2. Red de citaciones

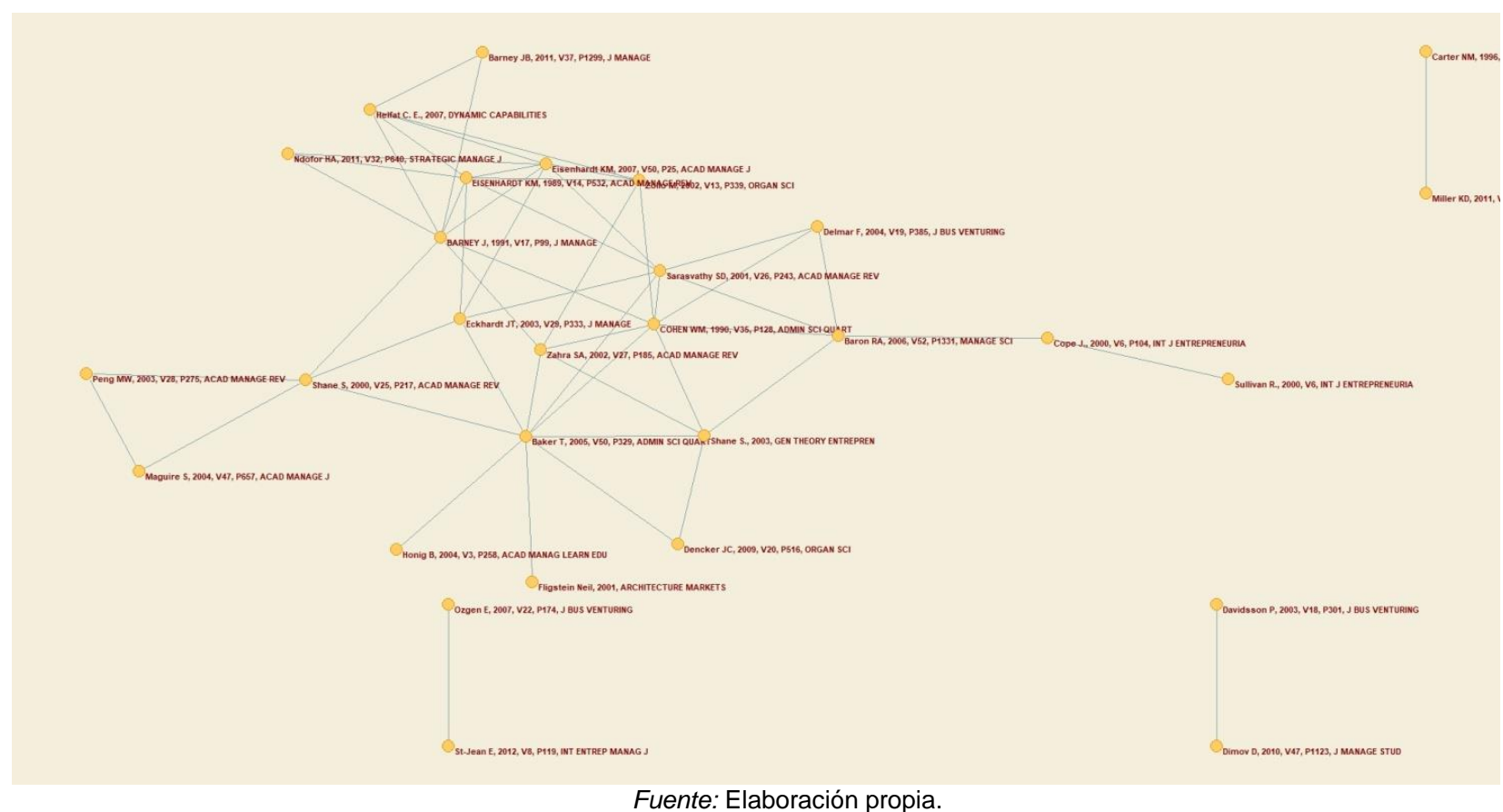




\section{FIGURA 3. Densidad de la red de citaciones}

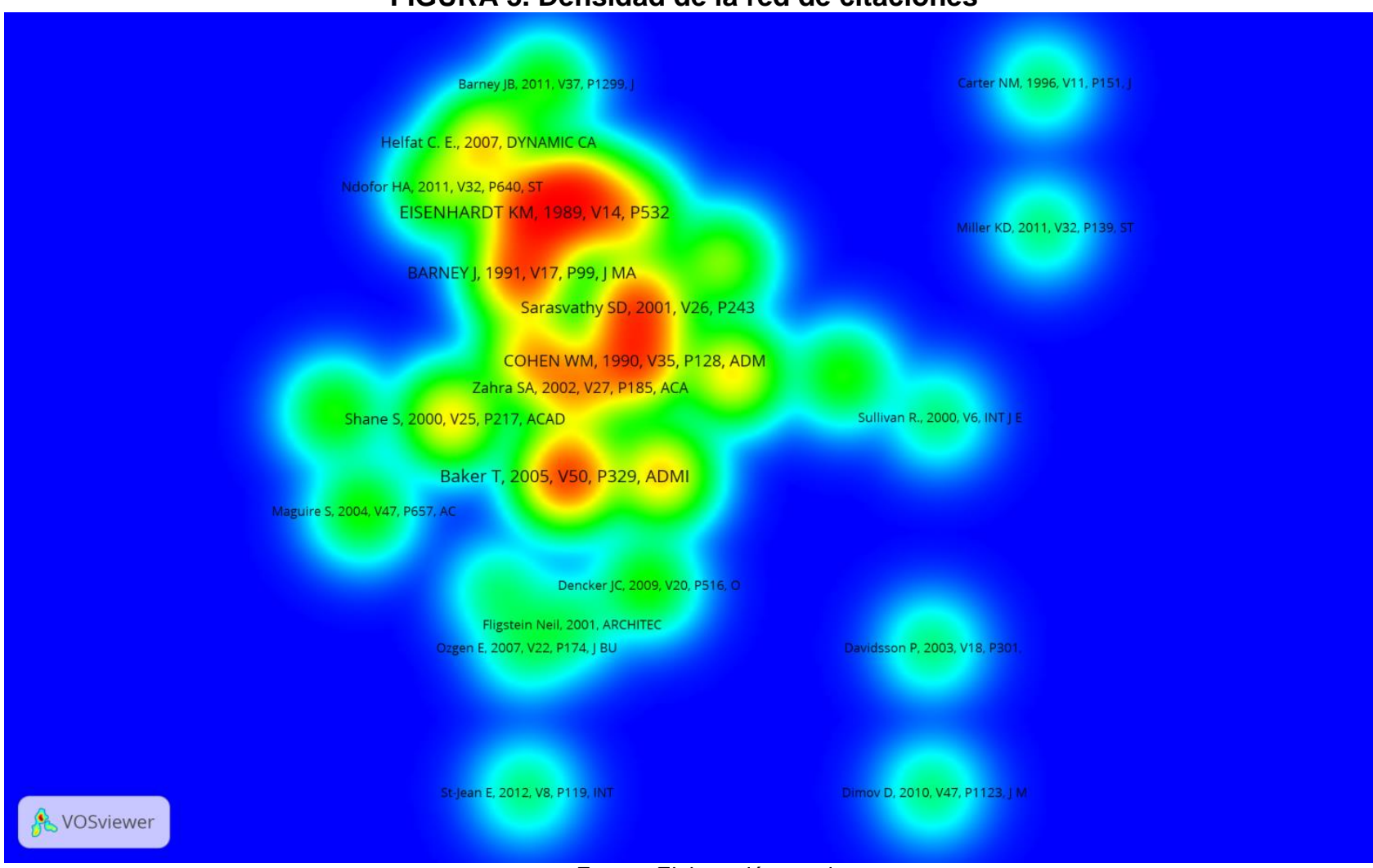

Fuente: Elaboración propia.

El análisis desarrollado pone en evidencia la escasa investigación sobre las necesidades formativas de los emprendedores y sobre la orientación para el emprendimiento. En términos cuantitativos los resultados muestran un insuficiente número de documentos científicos y citaciones para poder decir que se trata de un área de conocimiento visible. Incluso en las relaciones de colaboración se observan escasas interacciones entre investigadores. La escasa literatura sobre el tema es un aspecto limitante que no permite realizar discusión en profundidad sobre los resultados obtenidos. A continuación, se presenta la opinión de emprendedores noveles, manifiesta a través de un Delphi, que permitirá poner en contexto los resultados aquí expuestos.

\section{Requerimientos formativos de los emprendedores noveles: necesidades sentidas}

En este apartado se presentan las respuestas de las personas emprendedoras noveles en relación al valor de utilidad que otorgan a determinadas competencias, conocimientos y recursos necesarios para iniciar un proyecto emprendedor. Para definir el consenso se ha utilizado la regla aplicada en trabajos previos (Landeta, 2006; García-Aracil y Palomares-Montero, 2012): en una pregunta de dos respuestas alternativas un ítem acumula el $70 \%$ de las respuestas o en una pregunta de respuesta múltiple un ítem acumula al menos el $50 \%$ de las respuestas. Siendo, por tanto, el análisis de frecuencias el indicador de la respuesta individual que se expresa como porcentaje del total de frecuencias sobre el $100 \%$.

La Tabla 3 recoge la distribución porcentual de las respuestas de los emprendedores noveles con respecto a la utilidad que determinadas competencias tienen para iniciar un proyecto 
emprendedor. En general, se puede decir que el consenso alcanzado es considerable: en 24 de las 29 competencias presentadas, un ítem agrupa más del $50 \%$ de las respuestas. En todos los casos el consenso ha sido alcanzado para valorar como bastante o muy útil dichas competencias. El mayor consenso se observa en las competencias "capacidad de planificación y organización", "iniciativa" (metodológicas), y "tolerancia al fracaso" (de respuesta a contingencias, saber estar) con el $92.3 \%$ de las respuestas valorándolas como muy útiles. A continuación, les sigue la "toma de decisión" (metodológica), la "perseverancia" (de respuesta a contingencias, saber estar) y el "conocimiento del sector, del producto y/o servicio ofrecido por la empresa" (competencia profesional) con un $84.6 \%$ de las opiniones manifestando que son igualmente muy útiles. Por tanto, parece que los emprendedores noveles identifican necesarias competencias diversas para poder iniciar proyectos emprendedores.

Tabla 3. Opinión de emprendedores noveles sobre la utilidad de las competencias para iniciar un proyecto emprendedor (\%)

\begin{tabular}{|c|c|c|c|c|c|c|}
\hline $\begin{array}{l}\text { Tipo de } \\
\text { competencia }\end{array}$ & $\begin{array}{l}\text { Competencias necesarias para iniciar } \\
\text { un proyecto emprendedor }\end{array}$ & $\begin{array}{c}\text { Nada útil } \\
(1-2 \\
\text { puntos })\end{array}$ & $\begin{array}{l}\text { Poco útil } \\
\text { (3-4 } \\
\text { puntos) }\end{array}$ & $\begin{array}{l}\text { Algo útil } \\
\text { (5-6 } \\
\text { puntos) }\end{array}$ & $\begin{array}{l}\text { Bastante útil } \\
\text { (7-8 puntos) }\end{array}$ & $\begin{array}{l}\text { Muy útil } \\
\text { (9-10 } \\
\text { puntos) }\end{array}$ \\
\hline \multirow[t]{2}{*}{ Básicas } & $\begin{array}{l}\text { Comunicación (también idiomas } \\
\text { extranjeros) }\end{array}$ & - & 7.7 & 15.4 & 38.5 & 38.5 \\
\hline & Competencia informática & - & - & 7.7 & 38.5 & 53.8 \\
\hline \multirow{10}{*}{$\begin{array}{l}\text { Metodológicas } \\
\text { (saber hacer) }\end{array}$} & Autonomía & - & - & & 38.5 & 61.5 \\
\hline & Capacidad de análisis & - & - & 7.7 & 30.8 & 61.5 \\
\hline & Capacidad de aprendizaje & - & - & - & 23.1 & 76.9 \\
\hline & $\begin{array}{l}\text { Capacidad de planificación y } \\
\text { organización }\end{array}$ & - & - & 7.7 & - & 92.3 \\
\hline & Iniciativa & - & - & - & 7.7 & 92.3 \\
\hline & Innovación y Creatividad & - & - & - & 46.2 & 53.8 \\
\hline & Negociación & - & - & 7.7 & 46.2 & 46.2 \\
\hline & Pensamiento Crítico & - & - & 23.1 & 38.5 & 38.5 \\
\hline & Toma de decisiones & - & - & & 15.4 & 84.6 \\
\hline & Visión, sentido del propósito & - & - & 7.7 & 46.2 & 46.2 \\
\hline \multirow{9}{*}{$\begin{array}{l}\text { De relación y } \\
\text { cooperación con } \\
\text { el entorno } \\
\text { (saber ser) }\end{array}$} & $\begin{array}{l}\text { Gestión de conflictos/Resolución de } \\
\text { problemas }\end{array}$ & - & - & - & 38.5 & 61.5 \\
\hline & Habilidades de comunicación & - & - & - & 46.2 & 53.8 \\
\hline & Liderazgo & - & - & 7.7 & 30.8 & 61.5 \\
\hline & Trabajo en equipo/Cooperación & - & - & - & 23.1 & 76.9 \\
\hline & Autoconfianza & - & - & - & 23.1 & 76.9 \\
\hline & Motivación por el logro & - & 7.7 & 7.7 & 30.8 & 53.8 \\
\hline & Dinamismo y energía & - & - & 7.7 & 61.5 & 30.8 \\
\hline & Emprendimiento & - & - & - & 38.5 & 61.5 \\
\hline & Inteligencia emocional & - & - & - & 58.3 & 41.7 \\
\hline \multirow{6}{*}{$\begin{array}{l}\text { De respuesta a } \\
\text { contingencias } \\
\text { (saber estar) }\end{array}$} & Actitud positiva & - & - & 7.7 & 46.2 & 46.2 \\
\hline & Adaptabilidad & - & - & 7.7 & 30.8 & 61.5 \\
\hline & Perseverancia & - & - & - & 15.4 & 84.6 \\
\hline & Persuasión & - & - & 15.4 & 53.8 & 30.8 \\
\hline & Orientación al cliente & - & - & 7.7 & 23.1 & 69.2 \\
\hline & Tolerancia al fracaso & - & - & - & 7.7 & 92.3 \\
\hline
\end{tabular}




\begin{tabular}{|c|c|c|c|c|c|c|}
\hline $\begin{array}{l}\text { En gestión } \\
\text { empresarial }\end{array}$ & $\begin{array}{l}\text { Aspectos formales, legales, laborales, } \\
\text { de planificación...) }\end{array}$ & - & - & 25.0 & 50.0 & 25.0 \\
\hline Profesional & $\begin{array}{l}\text { Conocimiento del sector, del producto } \\
\text { y/o servicio ofrecido por la empresa }\end{array}$ & - & - & - & 15.4 & 84.6 \\
\hline
\end{tabular}

Fuente: Elaboración propia.

El grado de consenso disminuye en lo relativo a los conocimientos que se valoran útiles para iniciar un proyecto emprendedor (Tabla 4). En esta ocasión, el consenso es solo alcanzado en 9 de los 22 tipos de conocimientos propuestos. En general, se valoran útiles conocimientos relativos a la planificación estratégica ya que aspectos como "estrategias para obtener ventajas competitivas (diferenciación del producto, marketing)", "estrategias de crecimiento (marketing)", "estrategias de búsqueda de financiación", "plan económico financiero" y "plan de ventas" obtienen entre el $50 \%$ y el $70 \%$ de las respuestas para valorarlos como muy útiles. Lo mismo ocurre en el caso de los conocimientos relacionado con el análisis de mercado, identificando como muy útiles el "conocimiento/experiencia del sector/producto/servicio de la empresa/público objetivo" y el "conocimiento de metodologías de emprendimiento (start-up, design thinking)". En cambio, no hay consenso sobre la valoración que se puede realizar sobre el control de aspectos formales de la empresa o aspectos laborales relativos a los recursos humanos.

Tabla 4. Opinión de emprendedores noveles sobre la utilidad de los conocimientos requeridos para iniciar un proyecto emprendedor (\%)

\begin{tabular}{|c|c|c|c|c|c|c|}
\hline Tipo de Conocimiento & $\begin{array}{l}\text { Conocimientos requeridos para } \\
\text { iniciar un proyecto emprendedor }\end{array}$ & $\begin{array}{l}\text { Nada útil } \\
(1-2 \\
\text { puntos) }\end{array}$ & $\begin{array}{l}\text { Poco útil } \\
\text { (3-4 } \\
\text { puntos) }\end{array}$ & $\begin{array}{l}\text { Algo útil } \\
\text { (5-6 } \\
\text { puntos) }\end{array}$ & $\begin{array}{l}\text { Bastante } \\
\text { útil } \\
\text { (7-8 } \\
\text { puntos) } \\
\end{array}$ & $\begin{array}{l}\text { Muy útil } \\
\text { (9-10 } \\
\text { puntos) }\end{array}$ \\
\hline \multirow{3}{*}{$\begin{array}{l}\text { Aspectos formales de la } \\
\text { empresa }\end{array}$} & Formas jurídicas & - & 7.7 & 30.8 & 38.5 & 23.1 \\
\hline & Regímenes fiscales & - & 7.7 & 30.8 & 30.8 & 30.8 \\
\hline & Trámites de puesta en marcha & - & 7.7 & 46.2 & 15.4 & 30.8 \\
\hline \multirow{4}{*}{$\begin{array}{l}\text { Aspectos legales de la } \\
\text { empresa }\end{array}$} & $\begin{array}{l}\text { Legislación específica del producto } \\
\text { o servicio de la empresa }\end{array}$ & - & 7.7 & 30.8 & 30.8 & 30.8 \\
\hline & Legislación sobre riesgos laborales & - & 7.7 & 53.8 & 15.4 & 23.1 \\
\hline & Legislación sobre calidad & - & 15.4 & 38.5 & 30.8 & 15.4 \\
\hline & Legislación sobre medio ambiente & - & 15.4 & 38.5 & 38.5 & 7.7 \\
\hline \multirow{6}{*}{$\begin{array}{l}\text { Aspectos laborales } \\
\text { (Recursos humanos) }\end{array}$} & Modalidades de Contratación & - & 15.4 & 23.1 & 30.8 & 30.8 \\
\hline & Gestión de equipos & - & - & 7.7 & 46.2 & 46.2 \\
\hline & $\begin{array}{l}\text { Gestión de nóminas y seguros } \\
\text { sociales }\end{array}$ & - & 23.1 & 46.2 & 23.1 & 7.7 \\
\hline & Selección de personal & - & 7.7 & 15.4 & 30.8 & 46.2 \\
\hline & $\begin{array}{l}\text { Formación y orientación } \\
\text { profesional para el empleo }\end{array}$ & - & 23.1 & 23.1 & 46.2 & 7.7 \\
\hline & Obligaciones fiscales y mercantiles & - & 15.4 & 15.4 & 38.5 & 30.8 \\
\hline \multirow{4}{*}{ Planificación estratégica } & $\begin{array}{l}\text { Estrategias para obtener ventajas } \\
\text { competitivas (diferenciación del } \\
\text { producto, marketing) }\end{array}$ & - & 7.7 & - & 30.8 & 61.5 \\
\hline & $\begin{array}{l}\text { Estrategias de crecimiento } \\
\text { (marketing) }\end{array}$ & - & 7.7 & - & 38.5 & 53.8 \\
\hline & $\begin{array}{l}\text { Estrategias de búsqueda de } \\
\text { financiación }\end{array}$ & - & 7.7 & - & 38.5 & 53.8 \\
\hline & Plan económico financiero & - & 7.7 & - & 30.8 & 61.5 \\
\hline
\end{tabular}




\begin{tabular}{|c|c|c|c|c|c|c|}
\hline & Plan de ventas & - & 7.7 & - & 23.1 & 69.2 \\
\hline \multirow[b]{3}{*}{$\begin{array}{l}\text { Análisis del mercado } \\
\text { local, nacional y/o } \\
\text { internacional }\end{array}$} & Oferta y demanda & - & & 15.4 & 30.8 & 53.8 \\
\hline & $\begin{array}{l}\text { Principales competidores, } \\
\text { stakeholders (grupos de interés) }\end{array}$ & - & 7.7 & 7.7 & 38.5 & 46.2 \\
\hline & $\begin{array}{l}\text { Conocimiento/Experiencia del } \\
\text { sector/producto/servicio de la } \\
\text { empresa/público objetivo } \\
\text { Conocimiento de metodologías de } \\
\text { emprendimiento (start-up, design } \\
\text { thinking) }\end{array}$ & - & - & 8.3 & 33.3 & 66.7 \\
\hline
\end{tabular}

Fuente: Elaboración propia.

Las opiniones de los participantes en relación a la utilidad de determinados recursos para iniciar un proyecto emprendedor es más dispersa, a pesar de que el consenso es alcanzado en 9 de los 14 recursos valorados, aunque con porcentajes de respuesta, en general, más bajos que en los apartados anteriores (véase Tabla 5). Se consideran muy útiles aspectos como "Internet", la "formación", la "red de contactos (networking)" y los "recursos económicos (préstamos ICO, bonificación cuota autónomo, exenciones fiscales". Además, aparecen algunos recursos valorados como bastante útiles como los que puede ofrecer la "administración estatal", los "centros de iniciativas empresariales o viveros de empresa" o los "contacto con personas expertas en el ámbito de la empresa". En menor medida, las "Cámara de comercio" y la "consultoría de recursos humanos" son considerados recursos útiles para iniciar los proyectos emprendedores (51\% y 54\% respectivamente lo valoran como algo útiles).

Tabla 5. Opinión de emprendedores noveles sobre la utilidad de los recursos requeridos para iniciar un proyecto emprendedor (\%)

\begin{tabular}{|c|c|c|c|c|c|}
\hline $\begin{array}{l}\text { Recursos requeridos para iniciar un proyecto } \\
\text { emprendedor }\end{array}$ & $\begin{array}{c}\text { Nada útil } \\
\text { (1-2 } \\
\text { puntos) }\end{array}$ & $\begin{array}{c}\text { Poco útil } \\
\text { (3-4 } \\
\text { puntos) }\end{array}$ & $\begin{array}{c}\text { Algo útil } \\
\text { (5-6 } \\
\text { puntos) }\end{array}$ & $\begin{array}{c}\text { Bastante } \\
\text { útil (7-8 } \\
\text { puntos) }\end{array}$ & $\begin{array}{c}\text { Muy útil } \\
\text { (9-10 } \\
\text { puntos) }\end{array}$ \\
\hline Empleo & 7.7 & 7.7 & 38.5 & 38.5 & 7.7 \\
\hline Centros de apoyo al emprendimiento & 7.7 & - & 23.1 & 23.1 & 46.2 \\
\hline Administración estatal & 7.7 & - & 30.8 & 53.8 & 7.7 \\
\hline Unión Europea & 7.7 & 7.7 & 38.5 & 30.8 & 15.4 \\
\hline Ayuntamiento y/o sus Fundaciones & 7.7 & 7.7 & 23.1 & 46.2 & 15.4 \\
\hline Orientadores/as profesionales & 7.7 & - & 38.5 & 38.5 & 15.4 \\
\hline Cámara de comercio & - & 7.7 & 51.2 & 33.3 & 7.7 \\
\hline $\begin{array}{l}\text { Centros de iniciativas empresariales o viveros de } \\
\text { empresa }\end{array}$ & - & - & 33.3 & 50.0 & 16.7 \\
\hline $\begin{array}{l}\text { Contacto con personas expertas en el ámbito de la } \\
\text { empresa }\end{array}$ & - & - & - & 61.5 & 38.5 \\
\hline Internet & - & - & - & 38.5 & 61.5 \\
\hline Formación & - & - & - & 23.1 & 76.9 \\
\hline Red de contactos (networking) & - & - & - & 15.4 & 84.6 \\
\hline Consultoría de recursos humanos & 7.7 & - & 53.8 & 30.8 & 7.7 \\
\hline $\begin{array}{l}\text { Recursos económicos (préstamos ICO, } \\
\text { bonificación cuota autónomo, exenciones fiscales) }\end{array}$ & 7.7 & - & - & 25.0 & 58.9 \\
\hline
\end{tabular}

Fuente: Elaboración propia. 
Las opiniones vertidas por los emprendedores noveles participantes en el estudio evidencian la necesidad de formarse y potenciar aprendizajes en relación a competencias metodológicas, competencias de respuesta a contingencias (saber estar) y competencias profesionales. Sobre las dos primeras, los servicios de orientación profesional pueden contribuir enormemente al ampliar los saberes técnicos propios de la actividad profesional que se desea desarrollar. En este mismo sentido, la literatura revisada ha puesto de manifiesto la tradición de la academia por investigar con un enfoque de análisis de mercado también en la orientación profesional, hecho que queda corroborado en las opiniones de los participantes cuando subrayan la importancia de formarse en estrategias competitivas y de crecimiento, por ejemplo, pero no así sobre aspectos laborales o formales de la empresa.

\section{Conclusiones y discusión}

Reconociendo la limitación propia del criterio de búsqueda seleccionado en el análisis bibliométrico que excluye, por ejemplo, trabajos publicados en congresos internacionales, el estudio realizado en la primera fase de la investigación pone en evidencia la escasa literatura científica que se ha producido sobre el tema específico de la orientación profesional para la promoción del emprendimiento. Especialmente se puede resaltar este vacío en lo que respecta a estudios e investigaciones que avancen en la necesidad de realizar una orientación integral que supere la visión de la orientación hacia el emprendimiento centrada en el proyecto empresarial y su viabilidad económica. Pocos son los trabajos encontrados que desde una visión más educativa de la orientación propongan avances en el emprendimiento desde enfoques globalizadores que incluyan el proyecto vital. De hecho, como se ha podido observar, son muy pocas las revistas del área educativa las que han publicado artículos científicos sobre orientación profesional para el emprendimiento, mientras que destacan principalmente las revistas propias del área de la gestión y la economía.

Asimismo, a pesar de que se detecta un aumento en el número de trabajos científicos que aborden el tema objeto de estudio, se trata aún de una línea de investigación de corto recorrido que atiende aspectos de orientación profesional tradicionales y que parecen haber ayudado poco a encontrar las claves para la consolidación de los proyectos emprendedores nacientes o nuevos. De hecho, como informa GEM España (Global Enterpreneurships Monitor, 2017) del total de emprendedores nacientes (que paga salarios 3 o menos meses) y de emprendedores nuevos (que paga salarios entre 4 y 42 meses) (los cuales suponen, respectivamente, el $2.3 \%$ y el $2.9 \%$ de la población adulta entre 18 y 64 años) abandonan su proyecto empresarial tras el primer año un $25.8 \%$ de ellos. Se trata de un dato especialmente importante si se tiene en cuenta el hecho de que en tan solo un año, ciclo de vida de la empresa realmente corto, las personas emprendedoras noveles se ven involucradas en abandonar el proyecto empresarial. Algunos son los motivos que muestra el propio informe GEM España como por ejemplo la escasa rentabilidad, razones personales, problemas de financiación, entre otros. Desde el presente trabajo aventuramos a situar las causas de estas explicaciones, a tenor de las opiniones de los emprendedores noveles que han participado en el estudio Delphi desarrollado en la segunda fase de la investigación, en una falta de control sobre el propio contexto que acoge el proyecto emprendedor, por el escaso autoconocimiento personal y una limitada reflexión profunda del proyecto emprendido. 
De forma más concreta, los resultados mostrados con anterioridad en esta segunda fase del estudio permiten concluir que la orientación profesional para el emprendimiento identificada por el colectivo objeto de estudio (emprendedores noveles) no es considerada en atención a una visión integral. Se trata de sujetos más preocupados en formarse sobre conocimientos que requieren especialización en el área donde están emprendiendo (conocimiento del sector, producto 0 servicio; oferta y demanda, diferenciación del producto) y en temas financieros (plan económico y financiero, plan de ventas, búsqueda de financiación), obviando la necesidad de profundizar sobre cuestiones más internas a la propia persona como por ejemplo un autoconocimiento realista y positivo. A este respecto, estudios previos (Lucas Mangas y Sánchez García, 2017) señalan cómo emprendedores consolidados precisamente atribuyen un valor relevante a estos factores personales como aspectos clave para gestionar la conquista del mundo empresarial, siendo importantes también pero en menor medida variables relacionadas con el propio proyecto de emprendimiento.

Concluyendo, tanto la revisión de la literatura como las opiniones de las personas emprendedoras noveles muestran una escasa incidencia del papel que juega la orientación profesional en la construcción de un proyecto emprendedor entendida de la forma tradicional, próxima a una visión estrecha del proyecto empresarial, con foco principal en su viabilidad económica. No se puede evidenciar que desarrollar orientación profesional de modo más holístico, que atienda a aspectos personales y de proyecto de vida y articulados en coherencia con un proyecto profesional emprendedor, suponga mejorar las tasas o resultados de los proyectos emprendedores noveles pero, sin duda, los escasos trabajos científicos encontrados a este respecto animan a pensar que conviene profundizar sobre esta línea de investigación, proponiendo alternativas a los modelos formativos ya conocidos, abriendo diseños que motiven al emprendimiento con enfoques globalizadores. De hecho, las competencias y contenidos, en esencia, necesidades, que ponen encima de la mesa los/as emprendedores/as noveles delimitan un camino que permite encontrar las claves de esta formación y orientación desde el desarrollo personal y profesional en el marco de la gestión de la carrera. Como prospectiva al estudio aquí presentado, el equipo de investigación está trabajando en el diseño de un modelo formativo y orientador integrado que, con un estudio longitudinal, podrá testarse para conocer índices de éxito de los proyectos emprendedores iniciados tras la participación en este modelo formativo.

\section{Referencias Bibliográficas}

Baumen, Z. (1999). Modernidad líquida. Buenos Aires: Fondo de Cultura Económica.

Beck, U. (2006). Cosmopolitan Vision. Cambridge: Polity Press.

Bulmer, S. y Lequesne, C. (2013). The member states of the European Union. Oxford: Oxford University Press.

Cabero, J. y Infante, A. (2014). Empleo del método Delphi y su empleo en la investigación en comunicación y educación. EDUTEC, Revista Electrónica de Tecnología Educativa, 48, 1-16. DOI: http://dx.doi.org/10.21556/edutec.2014.48.187

Chisvert-Tarazona, M. J. (2014). Revisión del desarrollo de la orientación profesional como política $\begin{array}{llrrr}\text { activa de } & \text { empleo. } & & \end{array}$ DOI: https://doi.org/10.5944/reop.vol.25.num.1.2014.12010

Consejo Europeo (2000). Conclusiones del Consejo Europeo de Lisboa. Lisboa: Consejo Europeo. 
Cumming, D., Sapienza, H.J., Siegel, D.S., Wright, M. (2009). International entrepreneurship: managerial and policy implications. Strategic Entrepreneurship Journal, 3 (4), 283-296. DOI: $10.1002 /$ sej.75

Delgado López-Cózar, E. y Fernández Cano, A. (2002). El estudio de casos en las bases de datos del Science Citation Index, Social Science Citation Index y Arts and Humanities Citation Index (1992-2000). Arbor Ciencia, Pensamiento y Cultura, 171 (675), 609-629. DOI: https://doi.org/10.3989/arbor.2002.i675.1049

Dorta- González, P. y Ramírez-Sánchez, M. (2014). Producción e impacto de las instituciones española de investigación en Arts \& Humanitites Citation Index (2003-2012). Arbor Ciencia, Pensamiento y Cultura, 190 (770): a191. DOI: http://dx.doi.org/10.3989/arbor.2014.770n6012

Fairlie, R.W. y Robb, A.M. (2009). Gender differences in business performance: evidence from the Characteristics of Business Owners survey. Small Business Economics, 33 (4), 375-395. DOI: https://doi.org/10.1007/s11187-009-9207-5

García-Aracil, A. y Palomares-Montero, D. (2012). Indicadores para la evaluación de las instituciones universitarias: validación a través del método Delphi. Revista Española de Documentación Científica, 35 (1), 119-144. DOI: https://doi.org/10.3989/redc.2012.1.863

Giménez-Espert, M.C. y Prado-Gascó, V.J. (2018). Bibliometric analysis of six nursing journals from the Web of Science, 2012-2017. Journal of Advanced Nursing, 1-12. https://doi.org/10.1111/jan.13868

Global Enterpreneurships Monitor (2017). Informe GEM España 2016. Madrid: Editorial Universidad de Cantabria y Asociación RED GEM y CISE.

Grilo, I. y Irigoyen, J.M. (2006). Entrepreneurship in the EU: To Wish and not to be. Small Business Economics, 26 (4), 305-318. DOI: 10.1007/s11187-005-1561-3

Kumar, I.A. y Parveen, S. (2013). Teacher Education in the Age of Globalization. Research Journal of Educational Sciences, 1 (1), 8-12.

Landeta, J. (2002). El método Delphi: una técnica de previsión para la incertidumbre. Barcelona: Ariel.

Landeta, J. (2006). Current validity of the Delphi method in social sciences. Technological Forecasting and Social Change, 73 (5), 467-482. DOI: https://doi.org/10.1016/j.techfore.2005.09.002

Lucas-Mangas, S. y Sánchez-García, M. (2017). Competencias para el desarrollo de la carrera emprendedora: percepción de los profesionales desde un enfoque cualitativo. En: Suárez Ortega, M. Diseño de la carrera y gestión del talento emprendedor. Simposio 06 de AIDIPE 2017, Interdisciplinariedad y Transferencia.

Manso, J. y Thoilliez, B. (2015). La competencia emprendedora como tendencia educativa supranacional en la Unión Europea. Bordón, 67 (1), 85-99. DOI: https://doi.org/10.13042/Bordon.2015.67106

Morin, E., Ciurana, E.R. y Mota, R.D. (2002). Educar en la era planetaria. El pensamiento complejo como método de aprendizaje en el error y la incertidumbre humana. Valladolid: Universidad de Valladolid, Unesco, IIPC, USAL.

Palomares-Montero, D. y Chisvert-Tarazona, M.J. (2015). Do University Studies in Social and legal Field Teach Social Entrepreneur Competencies? Opción, 31 (1), 529-552.

Parlamento Europeo (2006). Decisión 1720/2006/CE por la que se establece un programa de acción en el ámbito del Aprendizaje Permanente. Diario Oficial de la Unión Europea, de 24 de noviembre de 2006, L, 327, 45-68. 
Parlamento y Consejo Europeo (2006). Recomendación 2006/962/CE del Parlamento Europeo y del Consejo, de 18 de diciembre de 2006, sobre las Competencias Clave para el aprendizaje permanente. Diario Oficial L 394 de 30.12.2006.

Patton, W. y McMahon, M. (2006). Career Development and Systems Theory. Connecting Theory and Practice. Rotterdam: SensePublishers.

Perren, L. y Jennings, P.L. (2005). Government discourses on entrepreneurship: issues of legitimization, subjugation and power. Entrepreneurship Theory and Practice, 29 (2), 173-184. DOI: 10.1111/j.1540-6520.2005.00075.x

Pfeilstetter, R. (2011). The entrepreneur. A critical reflection on the current uses and meanings of a concept. Gazeta de Antropologia, 27 (1).

Pinto, R. (2005). Challenges for public policy in promoting entrepreneurship in south Eastern Europe. Local Economy, 20 (1), 111-117. DOI: 10.1080/0269094042000326661

Sánchez-García, M.F. y Suárez-Ortega, M. (2017). Diseño y validación de un instrumento de evaluación de competencias para la gestión de la carrera emprendedora. Revista Iberoamericana de Diagnóstico y evaluación Psicológica, RIDEP, 45 (3), 109-123. DOI: https://doi.org/10.21865/RIDEP45.3.09

Savickas, M. (2005). The theory and practice of career construction. En BROWN, S. y LENT, R. (eds.), Career development and Counseling: Putting theory and research to work (42-70). Hoboken, NJ: Wiley.

Savickas, M. (2012). Life design: A paradigm for Career intervention in the $21^{\text {st }}$ Century. Journal of Counseling and Development, 90, 13-19. DOI: 10.1111/j.1556-6676.2012.00002.x

Savickas, M. (2013). The theory and practice of career construction. En S. Brown y R. Lent (eds.), Career development and counselling: Puttingtheory and research to work (147-183). Hoboken, $\mathrm{NJ}$ : Wiley.

Shane, S. y Venkataraman, S. (2000). The Promise of Empreneurship as a Field of Research. Academy of Management Review, 25 (1), 217-232. DOI: 10.2307/259271

Schoof, U. (2006). Stimulating youth entrepreneurship: barriers and incentives to enterprise startups by young people. Ginebra: International Labour Office.

Soto-González, M.D., Súarez-Ortega, M., Ortega-Leal, M.P. (en prensa). Referentes teóricos psicopedagógicos de la formación para el desarrollo de la carrera emprendedora. Revista Publicaciones.

Suárez-Ortega, M., Sánchez-García, M.F., García-García, M.C. (2016). Caracterización de buenas prácticas y necesidades de mejora en los servicios de orientación para el empleo. Revista Española de Orientación y Psicopedagogía, REOP, 27 (3), 43-60. DOI: https://doi.org/10.5944/reop.vol.27.num.3.2016.18799

\section{Fuentes electrónicas}

European Commission (2014). Employment and social affairs. Promoting jobs, inclusion and social policy as an investment. Recuperado el 13 de marzo de 2018, de: http://ec.europa.eu/social/BlobServlet?docld=10151\&langld=en 
OCDE (2001). Definition and Selection of Competencies: Theoretical and Conceptual Foundations (DeSeCo). Background Paper. Recuperado el 1 de enero de 2018, de: http://www.oecd.org/education/skills-beyond-school/41529556.pdf

Fecha de entrada: 30 de abril de 2018 Fecha de revisión: 7 de diciembre de 2018 Fecha de aceptación: 21 de enero de 2019 